\title{
Modified percutaneous radiologic gastrostomy technique without endoscopic or nasogastric access
}

\author{
Reda Tabashy ${ }^{1 *}$ (D) Amira Darwish ${ }^{2}$, Ashraf Ibrahim ${ }^{3}$ and Mohamed Gad El-Mola ${ }^{2}$
}

\begin{abstract}
Background: The aim of this study is to evaluate the efficacy and safety of a modified percutaneous radiologic gastrostomy (MPRG) technique under ultrasound and fluoroscopic guidance without endoscopic or nasogastric access.

Results: The study included 24 patients: 10 males and 14 females whose ages ranged from 44 to 80 years old. Ten patients had esophageal cancer and 14 patients had neck cancer. Technical success was achieved in 23 out of the 25 procedures (92\%). Two procedures failed (8\%) and were converted to the conventional technique by using the nasogastric tube. No major complications were reported. Minor complications were observed in 5 patients (20\%): intraperitoneal air and contrast leakage in 4 patients and focal mucosal dissection by the contrast in 1 patient.

Conclusion: The MPRG has high technical success rate, is safe with no major complications, and is most feasible when endoscopic or nasogastric access cannot be performed.
\end{abstract}

Keywords: Gastrostomy, Percutaneous gastrostomy, Stomach, Enteral feeding

\section{Background}

The majority of patients with advanced oral, pharyngeal, or esophageal cancers require nutritional support throughout their treatment period [1].

Chemotherapy and radiotherapy to the head and neck are associated with problems in swallowing, mucositis, and xerostomia. Also, the increased catabolic rate linked to malignancy further complicates weight loss and malnutrition, which can adversely affect outcome [2].

Enteral alimentation, regularly implemented by percutaneous gastrostomy (PG) and surgical jejunostomy, is superior to parenteral nutrition in terms of efficacy, morbidity, and cost-effectiveness [3].

Percutaneous gastrostomy is a non-surgical method of placing a feeding tube into the stomach through the anterior abdominal wall. Percutaneous endoscopic gastrostomy (PEG) and percutaneous radiologic gastrostomy (PRG) are the two techniques mostly used for the percutaneous tube placement [4].

\footnotetext{
* Correspondence: reda.tabashy@yahoo.com

${ }^{1}$ Department of Diagnostic and Interventional Radiology, National Cancer

Institute, Cairo University, Cairo 11796, Egypt

Full list of author information is available at the end of the article
}

Percutaneous endoscopic gastrostomy (PEG) has traditionally been the gold standard of gastrostomy tube insertion. It is widely available and relatively easy to perform, but high-grade narrowing or obstruction precludes the endoscopy passage [5]. In addition, there is a low but welldescribed risk of tumor seeding into the stoma, which is presumed to be a result of dissemination and implantation of tumor cells caused by the passage of the endoscope and/or the per-oral tube through the tumor tissue [6].

In the conventional PRG, the stomach is first distended with air through a nasogastric tube. In patients with tight pharyngeal or esophageal narrowing, coaxial guide wire system is used instead of the nasogastric tube to allow the introduction of small French catheter into the stomach through which air insufflation can be performed. But in patients with complete obstruction, even the passage of the nasogastric tube or the guide wire catheter system may not be possible [7].

The aim of our study is to evaluate the efficacy and safety of a modified percutaneous radiologic gastrostomy (MPRG) technique under ultrasound and fluoroscopic guidance without endoscopic or nasogastric access. 


\section{Methods}

\section{Patients}

Ethical approval to perform PRG at our institution was granted. Our study is a single-institution, prospective study of consecutive adult patients with upper digestive tract malignant obstruction, who underwent a modified gastrostomy technique between May 2013 and May 2014. During the study period, 24 consecutive patients were referred to the Interventional Radiology unit for the attempt of gastrostomy. Patients were provided with an informed consent about the procedure and the possible complications.

Inclusion criteria:

- Male or female patient 18 years and older.

- Consecutive adult patients suffering from dysphagia with need for enteral feeding.

- Ability to give informed consents for the study by patient or legal guardian.

Exclusion criteria:

- All current practice PRG contraindication [8].

- Acute gastrointestinal bleeding.

- Extremely obese patients (BMI > 40).

- Infectious disease local or systemic (e.g., sepsis, pancreatitis)

- Lack of a well-healed gastrostomy or infection around the insertion site.

\section{Procedure}

A total of 25 modified percutaneous radiologic gastrostomy (MPRG) procedures were attempted for all patients by an interventional radiologist (RT) with more than 20 years of experience in the field of interventional radiology and US of the abdomen. Twelve patients had history of failed attempt of PEG and/or conventional PRG because of the complete malignant upper digestive tract obstruction. One of those patients underwent esophageal stent that migrated downward and was obstructed, and the PEG could not be performed. Thirteen modified percutaneous radiologic gastrostomy (MPRG) techniques were performed for 12 patients without previous trial of endoscopic or conventional radiologic techniques. The modified technique was performed twice in one patient, after slippage of the first gastrostomy tube 1 month after its application.

\section{Patient's preparation and monitoring}

- Complete history was obtained from all patients, and appropriate physical examinations assured the suitability for gastrostomy (absence of abdominal hernias or cutaneous scars).
- The coagulation profile, complete blood picture, and the liver and the kidney profiles were checked. An international normalized ratio (INR) of less than 1.5 and a platelet count greater than $50000 \times 10^{9} / \mathrm{L}$

- All abdominal imaging studies were revised to exclude ascites or abdominal malignancies.

- The upper abdomen was prepped and dressed in the usual sterile manner.

- All patients were monitored for cardiac rhythm and rate, oxygen saturation, and blood pressure during the procedure.

- All patients received prophylactic intravenous antibiotics with cephalexin (Ceporex $1 \mathrm{~g}$; GSK) and antispasmodic hyoscine n-butyl bromide (Buscopan $10 \mathrm{mg}$; BoehringerIngelheim, Germany) at the start of the procedure.

- Intravenous sedation with $5 \mathrm{mg}$ midazolam (Dormicum, Roche, Basel, Switzerland) and $50 \mathrm{mg}$ pethidine (Demerol, Roche, Basel, Switzerland) were given to the patient.

\section{Gastric lumen anatomic delineation}

Ultrasound examination was performed by Acuson P500 ultrasound system (Siemens Healthcare $\mathrm{GmbH}$, Erlangen, Germany) on each patient to screen the abdomen for the presence of ascites or peritoneal malignancy and delineate the border of the left lobe of the liver by CH5-2 convex transducer, $1.4-5 \mathrm{MHz}$, to avoid its transgression during targeting the gastric lumen. Delineation of the anatomic position of the transverse colon was assessed fluoroscopically and by ultrasound.

The non-distended stomach was identified by the presence of a localized air collection in the epigastrium and the left subdiaphragmatic region. Ultrasound evaluation with superficial probe (VF10-5 linear transducer, 4.0-11.4 $\mathrm{MHz}$ ) was used to confirm the presence of a collapsed lumen in the epigastrium with the specific mural stratification, the gastric rugae, and any fluid contents inside.

\section{Gastric lumen access and inflation}

Local infiltration anesthesia at the decided entry site was administered. Under ultrasound guidance, the identified collapsed stomach was punctured using the 18-G needle of the loop-retained gastrostomy set (Tilma Ventricular Gastrostomy Set, Cook Medical) (Fig. 1a, Fig. 2a). Entry of the needle tip within the gastric cavity was confirmed by the injection of contrast medium through the connecting tube with opacification of the gastric rugae and by the passage of the contrast to the duodenum and jejunum (Fig. 1b, c, Fig. 2b, c).

The stomach was then inflated under fluoroscopic guidance with $600-800 \mathrm{~mL}$ of room air (Fig. 1d, Fig. 2c). Thereafter, a stiff guide wire was introduced into the 

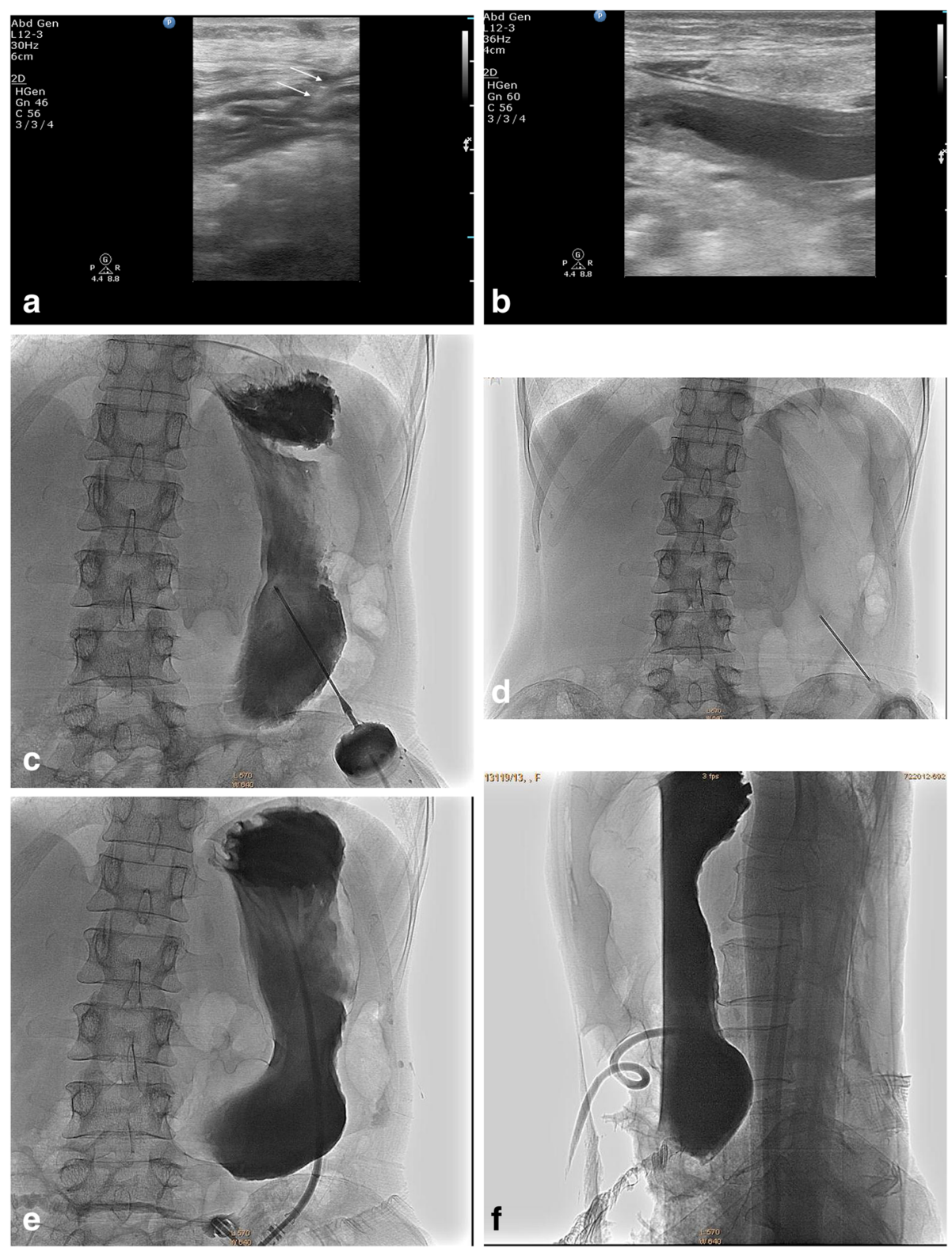

Fig. 1. A 53-year-old female patient with post-cricoid carcinoma. a Ultrasound-guided gastric access by 18-G needle (arrows). b Distension of the stomach by diluted non-ionic contrast medium. c Fluoroscopic confirmation of the intra-gastric position of the needle. $\mathbf{d}$ Gastric air insufflation through the needle. e Introduction of the peel-way sheath. $\mathbf{f}$ Lateral fluoroscopic view showing the gastrostomy tube in the final position

gastric lumen through the needle, which was then withdrawn (Fig. 2d).

\section{Gastrostomy tube insertion}

The peel-way sheath was advanced to the gastric lumen over the stiff guide wire, which was then removed with the trocar of the peel-way sheath (Fig. 2e). The gastrostomy tube then was introduced through the peel-way sheath, which was removed thereafter (Fig. 1f, Fig. 2e, f). The technical success was checked at the end of the procedure with $10 \mathrm{~mL}$ of contrast medium injected via the gastrostomy tube to confirm an intra-gastric position and to exclude extravasation or leakage. Finally, the gastrostomy tube was fixed to the skin.

\section{Aftercare}

All patients were admitted at the day care for a minimum of $6 \mathrm{~h}$ post-procedure. Early feeding $<4 \mathrm{~h}$ was started based on the meta-analysis that confirmed the safety of early feeding after PEG placement [9]. All complications related to PRG insertion were reported for a period of 3 months following the procedure. Complications of PRG 

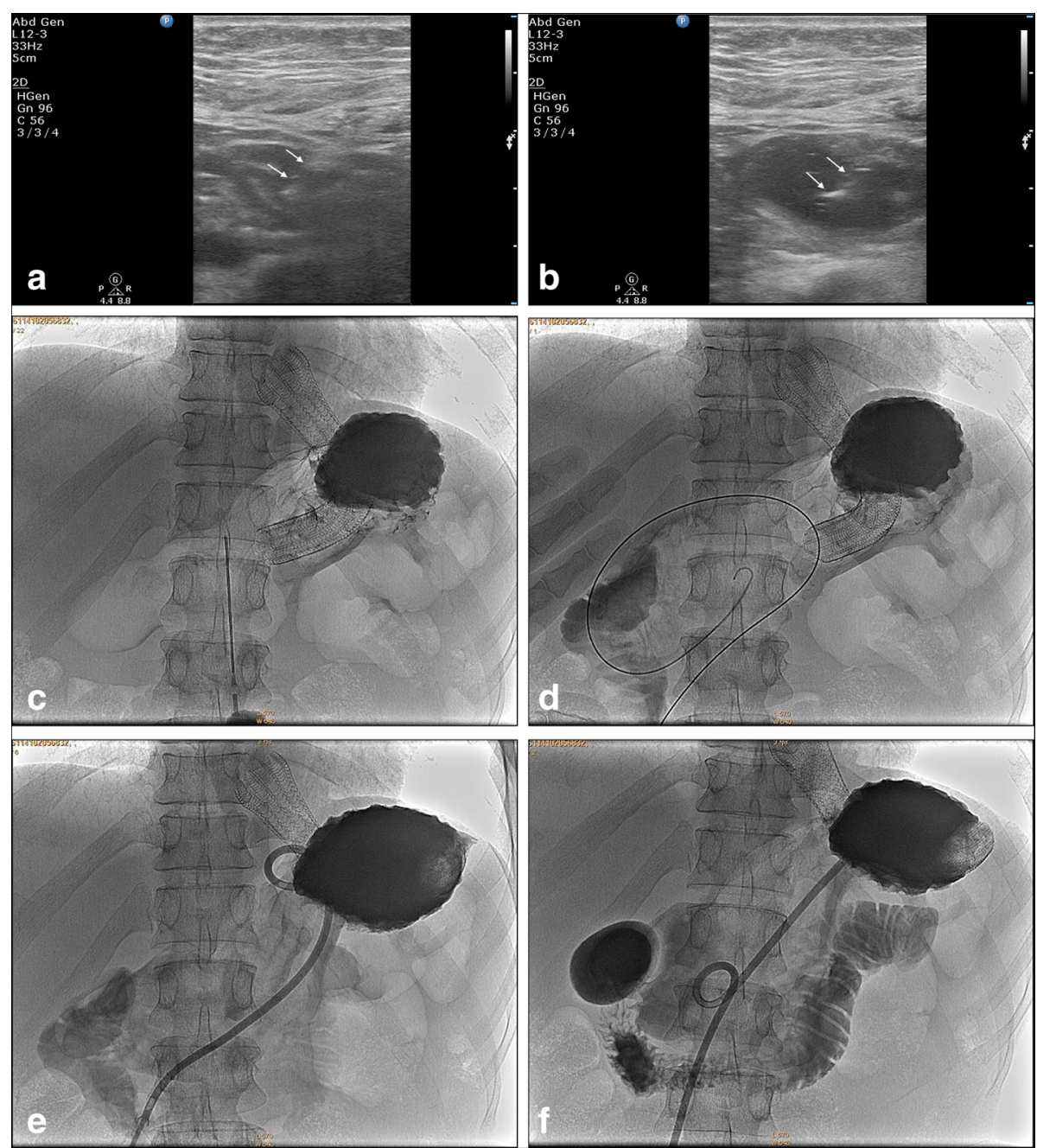

Fig. 2 A 45-year-old male patient with cancer esophagus and migrated non-functioning stent. a Ultrasound-guided gastric access by $18-G$ needle (arrows) in the antrum away from the migrated stent. b Distension of the stomach by diluted non-ionic contrast medium. c Fluoroscopic guided gastric insufflation after confirmation of the intra-gastric position of the needle. $\mathbf{d}$ Introduction of the stiff guidewire into the stomach. e Primary introduction of the gastrostomy tube into the stomach. $\mathbf{f}$ Final position of the gastrostomy tube within the stomach

tube placement are classified as minor or major according to the Society of Interventional Radiology (SIR) classification system for complications by outcome [10].

\section{Results}

During the study period, 24 patients with upper digestive tract malignant obstruction underwent a modified gastrostomy technique. Ten patients were males and 14 were females. Their ages ranged from 44 to 80 years old.

Ten patients ( 5 males, 5 females) had locally advanced esophageal, and 14 patients (5 males, 9 females) had neck cancers. Patients' characteristics are shown in Table 1.

Technical success was achieved in 23 out of the 25 procedures $(92 \%)$. Two procedures failed $(8 \%)$ due to failed gastric lumen access. Thus, conventional technique was applied in the same setting by using the nasogastric tube to distend the stomach by air, allowing anchoring of the anterior gastric wall to the anterior abdominal wall. This allowed easier gastric lumen access.

Minor complications were observed in 5 patients (20\%): intraperitoneal air and contrast leakage in 4 patients and focal mucosal dissection by the contrast in 1 patient. No major complications were encountered.

Our patients were followed up for 3 to 4 months after the procedure. The gastrostomy tube was slipped in one patient after 1 month from insertion. Re-insertion of another one was done by the same modified technique and followed for 3 months with no complications or slippage. No peritonitis or evidence of gastric leakage was noticed. 
Table 1 Characteristics of 24 patients with upper digestive tract malignant obstruction

\begin{tabular}{ll}
\hline Characteristic & Total (24 patients) \\
\hline Sex & 10 \\
Males & 14 \\
Females & $44-80$ \\
Age, year, range & Total (25 procedures) \\
Indications & 12 \\
Failed other gastrostomy techniques & 13 \\
Primary trial of the MPRG & \\
Primary tumor site & 10 \\
Esophageal cancer & 14 \\
Neck cancers & 1 \\
Tongue cancer & 3 \\
Laryngeal cancer & 2 \\
Post-cricoid cancer & 2 \\
Recurrent post-cricoid cancer & 3 \\
Thyroid cancer & 3 \\
Recurrent thyroid cancer &
\end{tabular}

\section{Discussion}

Gastrostomy is a commonly performed procedure to provide enteral nutrition in malnourished patients or at risk thereof. Clinical practice and techniques currently vary widely worldwide.

The success of PEG, conventional PRG, or CT-guided gastrostomy is often dependent on the presence of upper digestive tract sufficient caliber to allow passage of the endoscope, a nasogastric tube, or at least a guide wire-catheter assembly to provide access for optimal gastric inflation.

In our study, a modified PRG technique was attempted in which the ultrasound probe was used to localize the collapsed stomach and to guide the gastric access without the use of nasogastric tube.

Pugash and coworkers [11] reported on the feasibility of using ultrasound as an alternative mean for gaining access to the stomach after using effervescent to identify gastric bubble. DeBaere et al. [12] described a similar technique using sodium bicarbonate powder as an effervescent. Subsequently published studies advocated US for rapid puncture after filling the stomach with water through an NG tube which has no technical advantage over an NG tube inserted for air inflation [13, 14]. Thus, the single use of ultrasound has not become the standard technique in patients with upper digestive tract obstruction.

Quadri et al. [15] succeeded to undertake PRG safely in 9 (100\%) patients with complete obstruction of the upper digestive tract after failure of the conventional method. In 7 of the 9 patients (78\%), initial gastric puncture was achieved under direct US visualization. In the remaining 2 patients, gastric puncture was achieved under fluoroscopy due to presence of locules of gas in the stomach.

Chan and coworkers [7] described also a modified percutaneous radiologic gastrostomy technique in 14 patients with malignant pharyngeal (13 patients) and esophageal (1 patient) complete obstruction. They were unable to obtain PEG or the nasogastric tube for the conventional PRG. They suspected the position of the collapsed stomach by the presence of any loculated air at the left subdiaphragmatic region. They punctured the presumed collapsed stomach using a $21 \mathrm{G}$ under fluoroscopic guidance. Then, the needle was withdrawn gradually with continuous water-soluble contrast medium injection under fluoroscopic control until the intragastic position of the needle tip was confirmed by delineation of the gastric rugae by the contrast. This technique had high risk of retroperitoneal structures injury and peritoneal contrast leakage. To avoid this blind gastric access, we adopted the technique reported by Quadri et al. [15] and we used the linear ultrasound probe to localize the collapsed stomach and to guide the gastric access. This allowed us to avoid posterior gastric wall transgression, retroperitoneal structures injury, and contrast peritoneal leakage.

Chan and co-workers [7] used ultrasound only to delineate the margin of the left hepatic lobe, which is marked on the skin to avoid its injury. We did not perform this step as we actually used the ultrasound as a main guidance for the gastric access. When we confirmed by ultrasound that the needle was within the stomach, we continued the procedure as described by Chan and co-workers using the fluoroscopy to guide the remaining steps of the technique.

Ultrasound-guided access to the stomach had been previously described. Lorentzen et al. [16] evaluate the effectiveness and safety of PRG under US and fluoroscopic guidance provided the stomach can be properly distended with fluid through a nasogastric tube. The fluid-filled stomach was punctured under US guidance. Schlottmann and colleagues [17] evaluate the use of transabdominal ultrasound for PEG placement in patients in whom transillumination failed. Gastrostomies were satisfactory in 14 of 15 cases (93.3\%), and the complication rate was $28.5 \%$.

$\mathrm{Wu}$ and coworkers [18] investigate the utility of bedside US during replacement of malfunctioning or dislodged Gtube through the previously fashioned tract. After insertion, color Doppler was applied over the catheter tip to enhance visualization during gentle tube oscillation

Heberlein and coworkers [19] reported successful placement of the gastrostomy tube in 82 (96.5\%) out of the 85 patients without the use of nasogastric tube. Twenty-four patients had adequately distended stomach allowing fluoroscopic guided direct gastric puncture. The other 61 patients were given effervescent granules 
to distend the stomach before the puncture. The 3.5\% failure rate was due to inadequate gastric distension by the effervescent granules.

Inaba et al. [1] 2013 retrospectively investigated the medical records of 105 patients who underwent PRG. The technique used for PRG comprised insufflation to dilate the stomach via a nasogastric tube, followed by fluoroscopically guided puncture and gastrostomy tube placement. In patients for whom a nasogastric tube could not be inserted, the stomach was punctured with a fine needle under ultrasound-guidance and insufflated via this puncture needle to achieve dilation. The PRG procedure was successful in all cases including those who had undergone the modified technique.

Our technical success rate was $92 \%$ (23 out of 25 procedures) including 7 patients who had failed PEG and 5 patients who had failed PRG. This is similar to the success rates reported by Quadri et al. [15] and Chan et al. [7]. Our success rate also falls within the results reported in previous studies using conventional percutaneous radiologic gastrostomy [12, 20, 21].

The $20 \%$ rate of minor complications in our study was also low approaching the minimum accepted rate per SIR and American Gastroenterology Association guidelines (13-43\%) [22]. Our rate of leakage (16\%) was slightly higher than that with PEG but lower than that previously seen with RIG [23, 24]. No major complications were encountered. This is in contrast to the $6-7 \%$ rate of major complications reported by SIR [22].

Our study is limited by the small numbers included because of the low reference rate of patients with head and neck cancer with complete obstruction to our department during the study period. Furthermore, we did not compare the modified technique in our study to a group of patients to whom conventional technique was underwent. Further research in a randomized control fashion is warranted to optimize the procedure.

\section{Conclusion}

In conclusion, we proved with our experience of 24 patients with head, neck, and esophageal cancer that MPRG with direct gastric puncture using combined US and fluoroscopy-guided procedures is technically possible and safe with no major complications even in patients with complete upper digestive tract obstruction who failed PEG. Thus, this study is unique in that the MPRG overcomes the difficulty and even the unfeasibility of gastrostomy in patients with upper digestive tract complete obstruction. This technique can also be applied to those patients with incomplete upper digestive tract obstruction for whom NG tube insertion is potentially possible for conventional PRG to avoid both tumor bleeding and lengthy manipulations to pass the NG tube through the obstructed digestive tract segment.

\section{Abbreviations}

PEG: Percutaneous endoscopic gastrostomy; PRG: Percutaneous radiologic gastrostomy; NG: Nasogastric; MPRG: Modified percutaneous radiologic gastrostomy; SIR: Society of Interventional Radiology

\section{Acknowledgements \\ No acknowledgements.}

\section{Authors' contributions}

All authors contributed to the present study. RT and Al contributed to the conception and design. RT performed the technique and is responsible for the correspondence to journal. $\mathrm{AD}, \mathrm{MG}$, and $\mathrm{Al}$ collected and interpreted the data. AD was a major contributor in writing the manuscript. All authors contributed to the writing of the manuscript. All authors have read, revised, and approved the final manuscript.

\section{Funding}

No funding sources.

\section{Availability of data and materials}

The datasets used and/or analyzed during the current study are available from the corresponding author on reasonable request.

\section{Ethics approval and consent to participate}

This study was approved by the institutional review board of National Cancer Institute, Cairo University. The approval ID number is 201217120.3 and approval date is $12 / 2012$

Written informed consent was taken from all subjects who participated. Consent for publication

All patients included gave written informed consents to publish the data contained within this study.

\section{Competing interests}

The authors declare that they have no competing interests.

\section{Author details}

${ }^{1}$ Department of Diagnostic and Interventional Radiology, National Cancer Institute, Cairo University, Cairo 11796, Egypt. ${ }^{2}$ Department of Medical Oncology, National Cancer Institute, Cairo University, Cairo 11796, Egypt. ${ }^{3}$ Department of Surgical Oncology, National Cancer Institute, Cairo University, Cairo 11796, Egypt.

Received: 14 June 2019 Accepted: 27 November 2019

Published online: 06 December 2019

\section{References}

1. Inaba Y, Yamaura H, Sato Y, Kashima M, Kato M, Inoue D et al (2013) Percutaneous radiologic gastrostomy in patients with malignant pharyngoesophageal obstruction. Jpn J Clin Oncol. 43:713-718. https://doi. org/10.1093/jjco/hyt069

2. Mallinson PI, Tun JK, Byass OR, Cast JEl (2012) Usage of prophylactic radiologically inserted gastrostomy in head and neck cancer patients. Clin Radiol. 67:1175-1178

3. Bozzetti F, Braga M, Gianotti L, Gavazzi C, Mariani L (2001) Postoperative enteral versus parenteral nutrition in malnourished patients with gastrointestinal cancer: a randomised multicentre trial. Lancet Lond Engl. 358:1487-1492

4. Tamura A, Kato K, Suzuki M, Sone M, Tanaka R, Nakasato T et al (2016) CTguided percutaneous radiologic gastrostomy for patients with head and neck cancer: a retrospective evaluation in 177 patients. Cardiovasc Intervent Radiol. 39:271-278

5. Lowe AS, Laasch HU, Stephenson S, Butterfield C, Goodwin M, Kay CL et al (2012) Multicentre survey of radiologically inserted gastrostomy feeding tube (RIG) in the UK. Clin Radiol. 67:843-854

6. Cruz I, Mamel JJ, Brady PG, Cass-Garcia M (2005) Incidence of abdominal wall metastasis complicating PEG tube placement in untreated head and neck cancer. Gastrointest Endosc 62:708-711 quiz 752, 753

7. Chan S-C, Chu WC-W, Liu K-W, Liao C-T, Lee T-S, Ng S-H (2011) Modified radiology-guided percutaneous gastrostomy (MRPG) for patients with complete obstruction of the upper digestive tract and who are without endoscopic or nasogastric access. Korean J Radiol. 12:216. https://doi.org/10. 3348/kjr.2011.12.2.216 
8. Sutcliffe J, Wigham A, Mceniff N, Dvorak P, Crocetti L, Uberoi R (2016) CIRSE Standards of Practice Guidelines on Gastrostomy. Cardiovasc Intervent Radiol. 39:973-987

9. Bechtold ML, Matteson ML, Choudhary A, Puli SR, Jiang PP, Roy PK (2008) Early versus delayed feeding after placement of a percutaneous endoscopic gastrostomy: a meta-analysis. Am J Gastroenterol. 103:2919-2924

10. Cardella JF, Kundu S, Miller DL, Millward SF, Sacks D (2009) Society of Interventional Radiology. Society of Interventional Radiology clinical practice guidelines. J Vasc Interv Radiol JVIR 20(7 Suppl):S189-S191

11. Pugash RA, Brady AP, Isaacson S (1995) Ultrasound guidance in percutaneous gastrostomy and gastrojejunostomy. Can Assoc Radiol J J Assoc Can Radiol. 46:196-198

12. de Baere T, Chapot R, Kuoch V, Chevallier P, Delille JP, Domenge C et al (1999) Percutaneous gastrostomy with fluoroscopic guidance: single-center experience in 500 consecutive cancer patients. Radiology. 210:651-654

13. Lorentzen T, Skjoldbye B, Nolsøe C, Torp-Pedersen S, Mygind T (1995) Percutaneous gastrostomy guided by ultrasound and fluoroscopy. Acta Radiol Stockh Swed 1987 36:159-162

14. Bleck JS, Reiss B, Gebel M, Wagner S, Strassburg CP, Meier PN et al (1998) Percutaneous sonographic gastrostomy: method, indications, and problems. Am J Gastroenterol. 93:941-945

15. Quadri A, Umapathy N, Orme R (2005) Percutaneous gastrostomy in patients with complete obstruction of the upper digestive tract. Eur J Radiol. 56:74-77

16. Lorentzen T, Nolsøe CP, Adamsen S (2007) Percutaneous radiologic gastrostomy with a simplified gastropexy technique under ultrasonographic and fluoroscopic guidance: experience in 154 patients. Acta Radiol. 48:1319. https://doi.org/10.1080/02841850601045120

17. Schlottmann K, Klebl F, Wiest R, Grüne S, Kullmann F, Schölmerich J et al (2007) Ultrasound-guided percutaneous endoscopic gastrostomy in patients with negative diaphanoscopy. Endoscopy. 39:686-691. https://doi.org/10. 1055/s-2007-966604

18. Wu TS, Leech SJ, Rosenberg M, Huggins C, Papa L (2009) Ultrasound can accurately guide gastrostomy tube replacement and confirm proper tube placement at the bedside. J Emerg Med. 36:280-284

19. Heberlein WE, Goodwin WJ, Wood CE, Yousaf M, Culp WC (2012) Gastrostomy tube placement without nasogastric tube: a retrospective evaluation in 85 patients. Cardiovasc Intervent Radiol. 35:1433-1438

20. Given MF, Hanson JJ, Lee MJ (2005) Interventional radiology techniques for provision of enteral feeding. Cardiovasc Intervent Radiol. 28:692-703

21. Laasch H-U, Wilbraham L, Bullen K, Marriott A, Lawrance JA, Johnson RJ et al (2003) Gastrostomy insertion: comparing the options--PEG, RIG or PIG? Clin Radiol. 58:398-405

22. Itkin M, MH DL, Fang JC, SA MC, Kundu S, d'Othee BJ et al (2011) Multidisciplinary practical guidelines for gastrointestinal access for enteral nutrition and decompression from the Society of Interventional Radiology and American Gastroenterological Association (AGA) Institute, with endorsement by Canadian Interventional Radiological Association (CIRA) and Cardiovascular and Interventional Radiological Society of Europe (CIRSE). Gastroenterology 141:742-765. https://doi.org/10.1053/j.gastro.2011.06.001

23. McAllister $P$, Maclver $C$, Wales $C$, McMahon J, Devine JC, McHattie G et al (2013) Gastrostomy insertion in head and neck cancer patients: a 3 year review of insertion method and complication rates. Br J Oral Maxillofac Surg. 51:714-718. https://doi.org/10.1016/j.bjoms.2013.07.005

24. Silas AM, Pearce LF, Lestina LS, Grove MR, Tosteson A, Manganiello WD et al (2005) Percutaneous radiologic gastrostomy versus percutaneous endoscopic gastrostomy: a comparison of indications, complications and outcomes in 370 patients. Eur J Radiol. 56:84-90

\section{Publisher's Note}

Springer Nature remains neutral with regard to jurisdictional claims in published maps and institutional affiliations.

\section{Submit your manuscript to a SpringerOpen ${ }^{\circ}$ journal and benefit from:}

- Convenient online submission

- Rigorous peer review

- Open access: articles freely available online

- High visibility within the field

- Retaining the copyright to your article

Submit your next manuscript at $\boldsymbol{\nabla}$ springeropen.com 